\title{
CHROMATIC-CONTRAST THRESHOLD IMPAIRMENT IN DIABETES
}

\author{
S. J. TREGEAR ${ }^{1}$, P. J. KNOWLES ${ }^{2}$, L. G. RIPLEY ${ }^{3}$ and A. G. CASSWELL ${ }^{2}$ \\ Glasgow and Brighton
}

\begin{abstract}
SUMMARY
A prospective study was carried out to investigate acquired colour-vision deficits in diabetics using an automated, computer-controlled, cathode-ray-tube based test of chromatic contrast. Chromatic-contrast thresholds estimates were measured along both a red/ green (constant S-cone) confusion axis and a tritan (constant $M / L$-cone) confusion axis for 305 eyes of 305 diabetics. The diabetic data were partitioned into groups based on a clinical categorisation of retinopathy. The diabetic data were compared with both agematched and 'lens-equated' control data obtained from a bank of 347 normal subjects. Further analysis of differences between diabetic-status groups was performed. Associations between chromatic contrast threshold estimates and age, duration of disease, and severity of both macular oedema and ischaemia were investigated. The diabetic group was found to have significantly reduced chromatic-contrast threshold estimates when compared with normal controls, even in the absence of retinopathy. This reduction in chromatic contrast was predominantly tritanopic in nature. Interestingly, no reduction in red/green chromatic-contrast threshold estimate was found in diabetics without retinopathy. The tritan deficit seen in diabetics without retinopathy was strongly correlated with duration of disease, but when adjustments were made to account for the effects of duration-dependent lens yellowing, the tritan deficit was no longer apparent. A correlation between both the severity of macular oedema and severity of ischaemia with chromatic-contrast loss was established. Acquired reductions in both red/green and tritan chromatic-contrast threshold estimates seen in diabetics are strongly correlated with the severity of retinopathy. The results provide evidence that the specific tritan deficits seen in diabetics can be explained by the effects of lens yellowing rather than by selective
\end{abstract}

From: ${ }^{1}$ Glasgow Caledonian University, Cowcaddens Road, Glasgow; ${ }^{2}$ Sussex Eye Hospital, Eastern Road, Brighton, East Sussex; ${ }^{3}$ University of Sussex, Brighton, East Sussex, UK.

Correspondence to: S. J. Tregear, Department of Vision Science, Glasgow Caledonian University, Cowcaddens Road, Glasgow G4 0BA, UK. damage of the blue cone system as has been hypothesised by other groups. The results provide support for the potential use of automated CRT-based tests of colour vision in diabetic retinopathy screening protocols.

An association between diabetes and acquired colour-vision deficiency has been known to exist for more than 40 years. The earliest studies, however, were of small scale and did not examine the associations between fundus changes and colourvision deficits. ${ }^{1-6}$ The first published studies to demonstrate a relationship between diabetic retinopathy and colour-vision deficits were by Kinnear, Lakowski and Aspinall, ${ }^{7,8}$ who surveyed a population of 500 diabetics both with and without retinopathy using the Farnsworth-Munsell (FM) 100-hue test and the Pickford-Nicholson anomaloscope. They found that FM 100-hue scores were significantly poorer for diabetics than for normals and that the scores of those diabetics with retinopathy were significantly poorer than the scores of those diabetics without retinopathy. The losses seen within the diabetic group did not show any axis of colour-vision deterioration. Similar results were obtained with the Pickford-Nicholson anomaloscope except that the colour-vision deficits appeared to be of an acquired tritanopic type. Since then, other studies have convincingly demonstrated a correlation between colour-vision loss, especially tritan-type loss, and diabetic retinopathy. ${ }^{-15}$

Reffin ${ }^{16}$ and de Alwis ${ }^{17}$ recently investigated the relationship between fundus features and colourvision deficits in 137 diabetic subjects using an automated CRT-based test of colour vision performed with the Sussex Gratings Machine (SGM). The SGM test, like other CRT-based colour-vision tests, has many advantages over more traditional methods of colour-vision testing used in clinical research, such as the Farnsworth-Munsell 100-hue test. It allows precise measurement of colour-vision performance along specific chromatic confusion axes, 
is quick and easy to perform and to score, and does not suffer from problems of unsuitable illumination or pigment degeneration.

The SGM produces an equiluminant, sinusoidal, chromatic grating on a high-resolution colour monitor. The chromaticity of the sinusoid can be changed along a red/green (constant S-cone) confusion axis or along a tritan (constant $\mathrm{M} / \mathrm{L}$-cone) confusion axis about a white point. The maximum amplitude of the chromatic excursion about the white point is changed under programmed control and the value at which a subject just perceives coloured stripes is found using an interleaved double-staircase reversal method. This value is taken to be the chromatic-contrast threshold estimate for that particular opponentprocess system and it is with these two figures, one for red/green contrast threshold and one for tritan contrast threshold, that we have categorised colour vision in the subjects discussed in this paper.

Using the SGM system, Reffin and de Alwis found that age, duration of disease, the degree of macular oedema and the degree of ischaemia all had a significant correlation with colour vision. Interestingly, their study also showed that some of the fundus features that are used clinically to determine whether laser photocoagulation is indicated, such as the presence of hard exudates or microaneurysms within $500 \mu \mathrm{m}$ of the macula, or the presence of new vessels, had no significant correlation with colour vision.

In this paper we report on further work, also carried out using the SGM system, on the investigation of acquired colour-vision deficiencies found in diabetic subjects. The work was carried out at the Sussex Eye Hospital, Brighton, over a 3 year period and this paper summarises the results and conclusions drawn from the chromatic-contrast data obtained from 305 diabetic subjects. Three main areas of interest concerning the relationship between acquired colour-vision loss and diabetes are examined in this paper. Firstly, the relationship between clinical classification of diabetic retinopathy and colour vision is investigated. Secondly, the effects of age and of duration of disease on colour vision are examined. Finally, the effects of macular oedema and ischaemia on colour vision are considered.

\section{SUBJECTS AND METHODS}

\section{Subjects}

Chromatic-contrast-threshold data obtained from 305 randomly chosen eyes of 305 diabetic subjects were included in this study (mean age $=56$ years (range 17-88 years), mean duration of disease $=14$ years (range 1.5-60 years)). The subjects consisted of 92 type I diabetics and 213 type II diabetics. All the subjects were consenting volunteers recruited at the Sussex Eye Hospital, Brighton, or via the diabetic photographic clinic at the Royal Sussex County Hospital, Brighton. Exclusion criteria included previous laser treatment, signs of significant lens opacification as determined by slit-lamp examination through dilated pupils, or a corrected visual acuity of worse than 6/18. Since one of the ultimate aims of this work was to develop a test with which to aid in screening the diabetic population for sight-threatening diabetic retinopathy we have deliberately not excluded those diabetics who are taking any form of medication other than those used to control glucose levels. It is well known that some medications will affect colour vision but we decided that a screener should be tested on diabetics who are as representative as possible of the diabetic population. Many diabetics, especially in later life, will be on some form of medication.

\section{Classification of Diabetic Retinopathy}

Following assessment of Snellen visual acuity and chromatic contrast, each diabetic eye was dilated with $1.0 \%$ tropicamide. Fundal examination was then carried out by an ophthalmologist experienced in diabetic-retinopathy classification using a slit-lamp biomicroscope and a 78-dioptre lens. Each eye was categorised depending on its stage of retinopathy based on the following clinically orientated system:

No retinopathy: No evidence of retinopathy can be seen clinically.

Background: Characterised by the development of microaneurysms; superficial and deep retinal haemorrhages; the formation of hard exudates.

Pre-proliferative: Characterised by five or more cotton-wool spots on the fundus; the presence of white vessels, venous bleeding and venous loops; features associated with severe background retinopathy such as widespread blotchy dark haemorrhages.

Proliferative: Characterised by the presence of new blood vessels: at the optic disc; elsewhere on the retina if more than half a disc diameter in size; less than half a disc diameter if associated with vitreous haemorrhage; or by vitreous haemorrhage anywhere. Maculopathy: Clinically significant macular oedema as characterised by the ETDRS group. ${ }^{18.19}$ Characterised by the presence of a thickening of the retina within $500 \mu \mathrm{m}$ of the centre of the macula; presence of hard exudates associated with retinal thickening within $500 \mu \mathrm{m}$ of the centre of the macula; a zone of retinal thickening one disc area or larger in size within a disc diameter of the centre of the macula.

The ophthalmologist making the categorisation had no knowledge of the results obtained from the chromatic-contrast test, which had been carried out prior to the clinical assessment. 
Table I. Summary of the data obtained from the 305 eyes of 305 diabetic patients included in this study. The data have been partitioned into diabetic retinopathy category (see text)

\begin{tabular}{lccccc}
\hline & $\begin{array}{c}\text { No retinopathy } \\
(n=87)\end{array}$ & $\begin{array}{c}\text { Background retinopathy } \\
(n=116)\end{array}$ & $\begin{array}{c}\text { Maculopathy } \\
(n=63)\end{array}$ & $\begin{array}{c}\text { Pre-proliferative retinopathy } \\
(n=26)\end{array}$ & $\begin{array}{c}\text { Proliferative retinopathy } \\
(n=13)\end{array}$ \\
\hline TCT & 0.55 & 0.74 & 1.14 & 1.05 & 1.13 \\
& $(0.17)$ & $(0.29)$ & $(0.31)$ & $(0.26)$ & $(0.31)$ \\
RGCT & 0.45 & 0.57 & 0.81 & $(0.73$ & 0.88 \\
& $(0.17)$ & $(0.24)$ & 62.32 & 63.04 & $(0.34)$ \\
Age & 52.09 & 55.10 & $(11.94)$ & $(14.39)$ & $(18.92$ \\
& $(16.89)$ & $(14.95)$ & & \\
\hline
\end{tabular}

\section{Test Procedure}

The SGM has been described in detail elsewhere and is essentially a colour-vision testing system designed to screen for, and quantify, acquired colour-vision deficiencies in a clinical situation. ${ }^{16,17,20}$ The SGM was set up to produce vertical, low-spatial-frequency, equiluminant, sinusoidal gratings on a high-resolution colour monitor (mean luminance $20 \mathrm{~cd} \mathrm{~m}^{-2}$ ) which were randomly tritan or $\mathrm{red} / \mathrm{green}$. The chromaticity of the gratings was changed along either a tritan or red/green confusion axis about the equal energy, neutral white point (CIE $1976 u^{\prime}, v^{\prime}$, coordinates: $0.21,0.47$ ). The maximum excursion of the chromatic grating about the white point was changed under computer-program control using a double-staircase reversal method ${ }^{21}$ and the value at which the subject just perceived coloured stripes was found for each stimulus. These values were taken to be the tritan-contrast threshold (TCT) estimate and the red/green-contrast threshold (RGCT) estimate respectively, and it was with these measures that we categorised colour-vision deficits. Since TCT and RGCT estimates are thresholds, it should be noted that an increase in either will denote a decrease in the respective opponent-process channel integrity.

Each subject was positioned $2 \mathrm{~m}$ from the CRT display so that the stimuli subtended a visual angle of $4^{\circ}$. At this distance the gratings produced on the CRT had a spatial frequency of 0.66 cycles/deg. Having been randomly chosen by the throw of a coin, one of the subject's eyes was occluded and the subject was instructed to look at the CRT screen. If, following an auditory warning tone, the subject saw coloured stripes, a hand-held button was to be pressed. If the subject did not see any stripes, or was unsure, the button was not to be pressed. As this test is a psychophysical test, it was very important that identical instructions were given to each subject to ensure that they employed the same criteria when deciding whether or not to press the button. To ensure that the subject understood the procedure correctly, one manually controlled test trial was carried out. The test proper was then commenced under automated computer control and ended after five double-staircase reversals had been completed for both the tritan and red/green stimuli. Chromaticcontrast threshold estimates were determined based on the mean of only the last four reversals. The TCT and RGCT data for each eye were stored in the computer database, along with all the subjects' details, for recall and analysis at a later date.

\section{RESULTS}

The diabetic data set was partitioned into groups according to the ophthalmic categorisation of retinopathy. Both TCT and RGCT data distributions for each of the five retinopathy categories were tested for normality using the K-S (Lilliefors) normality test. All the data sets were found to be normally distributed. The mean and standard deviation of each data set are summarised in Table I. Paired $t$-tests confirmed that TCT estimates were significantly poorer than RGCT estimates in all retinopathy groups (Table II). A one-way ANOVA established that statistically significant differences existed between some of the retinopathy categories for both the TCT and RGCT data (TCT: $F$ ratio $=$ 53.16; $p<0.0001$; RGCT: $F$ ratio $=28.25, p<0.0001)$. Inter-group comparisons were made using the Scheffé procedure with the significance level set at $p \leqslant 0.05$; the results are shown in Table III.

Table II. The results of $t$-tests used to determine whether a statistically significant difference existed between the RGCT and TCT data within each diabetic retinopathy categorisation group

\begin{tabular}{lccccc}
\hline & No retinopathy & Background retinopathy & Maculopathy & Pre-proliferative retinopathy & Proliferative retinopathy \\
\hline RGCT & 0.45 & 0.57 & 0.81 & 0.73 & 0.88 \\
$\quad$ Mean & & & & & \\
$\quad$ Variance & 0.03 & 0.06 & 0.08 & 0.08 & 0.12 \\
TCT & 0.55 & 0.74 & 1.14 & 1.05 & 1.13 \\
Mean & & 0.09 & 0.09 & 0.07 & 0.09 \\
Variance & 0.05 & 9.30 & 13.04 & 8.14 & 4.80 \\
$t$ & 5.39 & $<0.0001$ & $<0.0001$ & $<0.0001$ & $<0.0005$ \\
$p$ & $<0.0001$ &
\end{tabular}

It can be seen that in all retinopathy groups the TCT data are significantly elevated when compared with the RGCT data. This indicates that tritan colour vision is more severely impaired by diabetic retinopathy than red/green colour vision. 
Table III. Results of the Scheffé inter-group comparison test for both RGCT data and TCT data

\begin{tabular}{|c|c|c|c|c|c|}
\hline & No retinopathy & $\begin{array}{l}\text { Background } \\
\text { retinopathy }\end{array}$ & Maculopathy & $\begin{array}{l}\text { Pre-proliferative } \\
\text { retinopathy }\end{array}$ & $\begin{array}{l}\text { Proliferative } \\
\text { retinopathy }\end{array}$ \\
\hline \multicolumn{6}{|l|}{$R G C T$ data } \\
\hline No retinopathy & & * & * & * & * \\
\hline Background retinopathy & * & & * & * & * \\
\hline Maculopathy & $*$ & * & & * & \\
\hline Pre-proliferative retinopathy & * & * & * & & \\
\hline Proliferative retinopathy & $*$ & $*$ & & & \\
\hline \multicolumn{6}{|l|}{ TCT data } \\
\hline No retinopathy & & * & * & * & * \\
\hline Background retinopathy & * & & * & * & * \\
\hline Maculopathy & * & * & & & \\
\hline Pre-proliferative retinopathy & * & * & & & \\
\hline Proliferative retinopathy & * & * & & & \\
\hline
\end{tabular}

This test was only performed if the one-way ANOVA showed that a significant inter-group difference existed. The asterisk indicates that a significant inter-group difference exists at the $p<0.05$ level.

Fig. 1 shows a scatterplot of both TCT data and RGCT data versus age for those diabetic eyes classified as having no retinopathy. The continuous curves represent the standard deviation limit of normality derived from data obtained from 347 normal eyes. A two-sample $t$-test found that no significant difference in RGCT existed between diabetic eyes with no retinopathy $(n=87)$ and an age-matched control group $(t=0.95, \quad p=0.345)$. However, TCT estimates were found to be significantly elevated in the no-retinopathy group compared with the same control group $(t=2.72$, $p<0.001)$.

A regression analysis of the relationship between age and RGCT in both the no-retinopathy group and the corresponding age-matched control group

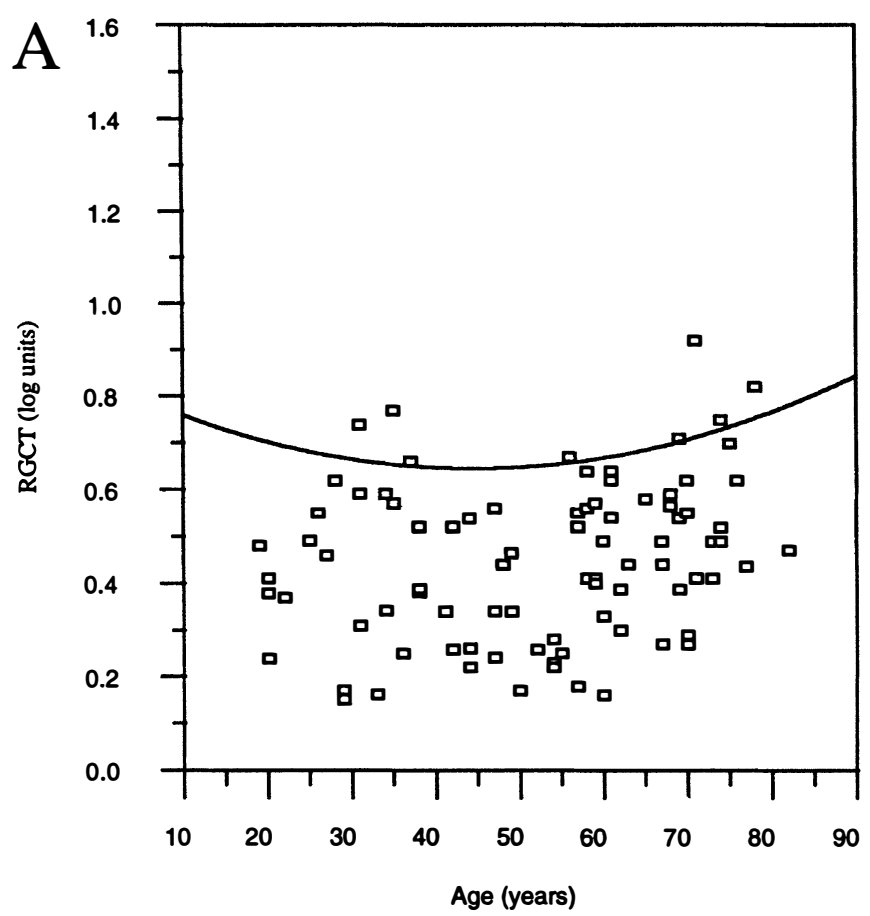

showed that no significant increase in RGCT was associated with increasing age in either group (noretinopathy group: $t=2.01, p=0.0529$; controls: $t=0.645 ; p=0.523)$. However, this was not the case when a regression analysis of TCT versus age was performed for both groups. It turned out that the TCT data from the control group showed no significant increase with age $(t=1.200, p=0.240)$, whereas the TCT data from the no-retinopathy group $\operatorname{did}(t=3.463, p<0.005)$.

The effects of age were analysed more closely for the no-retinopathy group by partitioning the age variable into its constituents: age at onset of diabetes; duration of diabetes. Since duration cannot be estimated accurately for type II diabetics, only type I diabetics were considered in this analysis. Multiple-

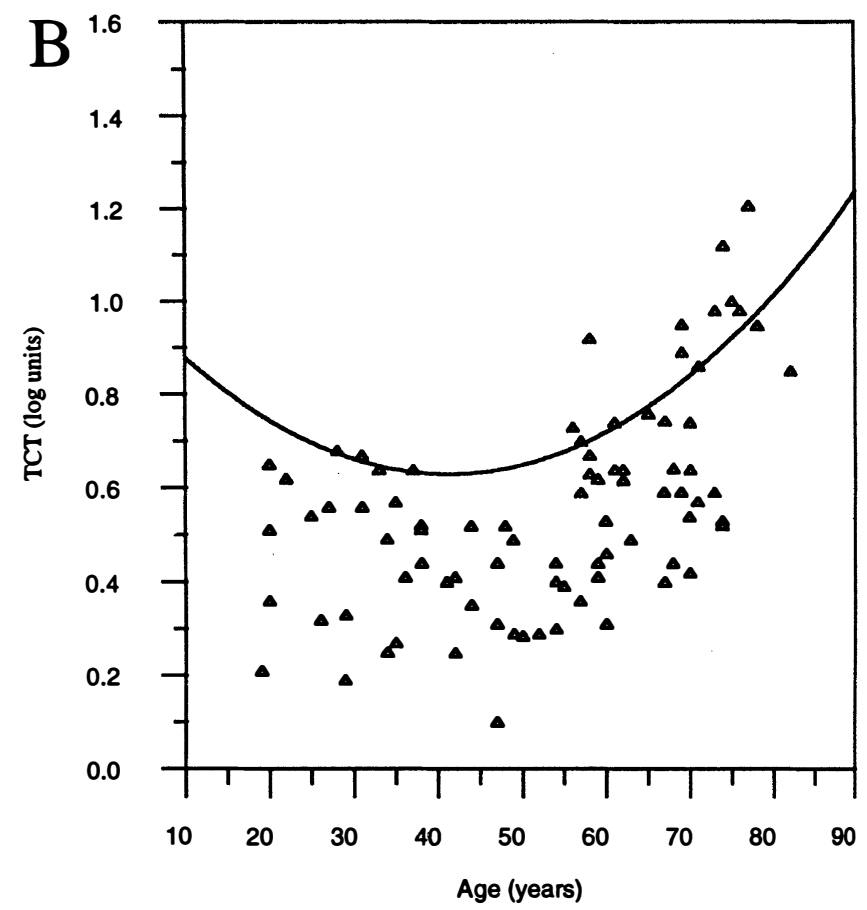

Fig. 1. Scatterplots for both RCGT $(A)$ and TCT $(B)$ estimates obtained from 87 diabetic eyes classified as having no diabetic retinopathy plotted against age. The continuous curve represents the respective 2 standard deviation limit of normality. Estimates which fall below the curve should be considered as normal. 
regression analysis found that no significant relationship existed between age at onset, or duration, and RGCT in diabetics with no retinopathy (ANOVA: $F$ ratio $=2.53, p=0.10)$. However, a significant relationship was found to exist between age at onset or duration and TCT (ANOVA: $F$ ratio $=$ $8.81, p<0.001)$. Further analysis showed that duration was significantly associated with an increase in TCT $(t=4.188, p<0.0005)$ but age at onset was not $(t=0.461, p=0.6476)$.

These results indicate that the increase in TCT seen in type I diabetics with no retinopathy can be attributed to duration-dependent effects and might be caused by the development of microscopic diabetes-induced changes in the retina which selectively affect the tritan colour-vision system. Alternatively, it is possible that the increase in TCT is in part, if not completely, due to pre-retinal absorption of short-wavelength light resulting from lens yellowing. $^{22}$ In order to investigate this possibility, a comparison was carried out between type I diabetics with no retinopathy and a group of 'lens-equated' controls.

The rate of lens yellowing in diabetics has been shown to be linearly related to diabetic duration. ${ }^{22}$ Moreland ${ }^{23}$ has derived two simple equations (Equations 1 and 2) which relate Lutze and Bresnick's ${ }^{22}$ linear lens yellowing model with Pokorny et al.'s ${ }^{24}$ linear model of normal lens 'ageing':

$$
\begin{aligned}
& E=A+2.54 T-3.8 \\
& E=0.30 A+0.76 T+40.9
\end{aligned}
$$

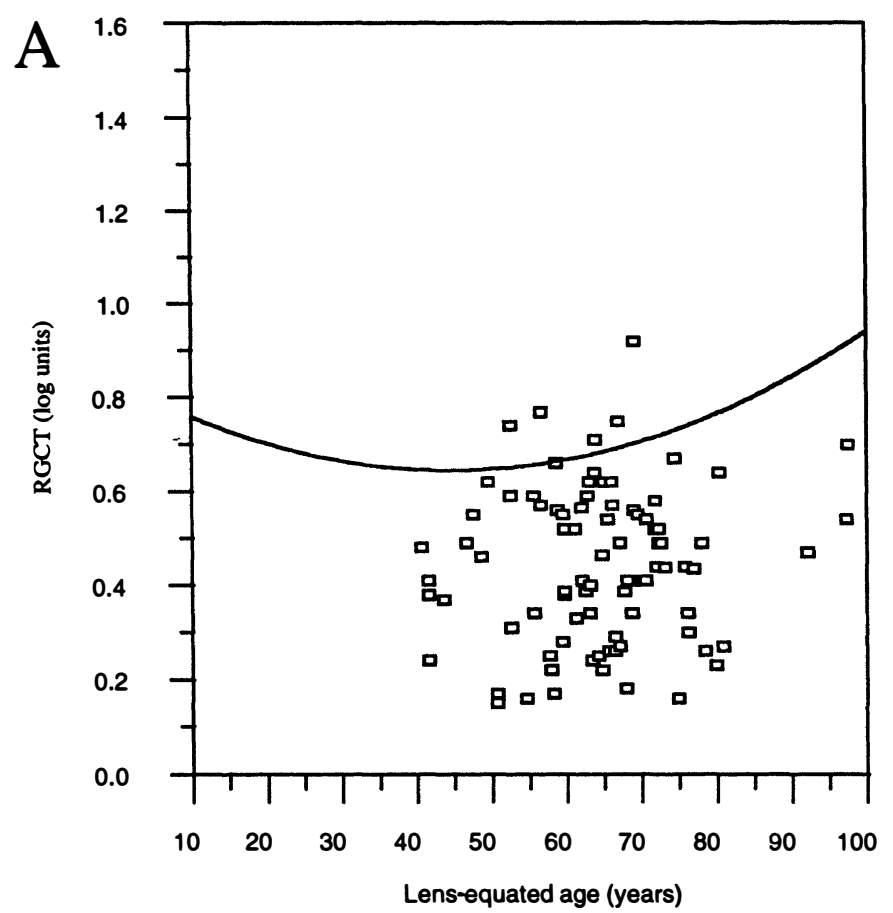

where $E$ is the age of a normal subject having the same lens-absorption characteristics as a diabetic, $A$ is the age of the diabetic and $T$ is the duration of the diabetes, which must be greater than 1.5 years. Equation (1) is used when $E$ is greater than 20 but less than 60. Equation (2) is used when $E$, derived from Equation (1), is greater than 60 .

As an example of the use of these equations, consider two diabetics both aged 35 years. Diabetic A has suffered from diabetes for 3 years whereas diabetic $\mathrm{B}$ has suffered for 22 years. For diabetic A, apply Equation (1) with $A=35$ and $T=3$ :

$$
\begin{aligned}
E & =A+2.54 T-3.8 \\
& =35+7.6-3.8=38.8
\end{aligned}
$$

For diabetic B apply Equation (1) with $A=35$ and $T=22$ :

$$
\begin{aligned}
E & =A+2.54 T 23.8 \\
& =35+55.9-3.8=87.1
\end{aligned}
$$

Since $E$ is greater than 60 then apply Equation (2) with $A=35$ and $T=22$ :

$$
\begin{aligned}
E & =0.30 \mathrm{~A}+0.76 T+40.9 \\
& =10.5+16.7+40.9=68.1
\end{aligned}
$$

Thus diabetic A would be compared with a normal subject of age 39 years, whereas diabetic B would be compared with a normal subject of age 68 years.

The lens-equated age of each diabetic was determined using the Moreland technique described above and the appropriate 'lens-equated' control data were then selected from the normal control database. Fig. 2 shows both TCT and RGCT

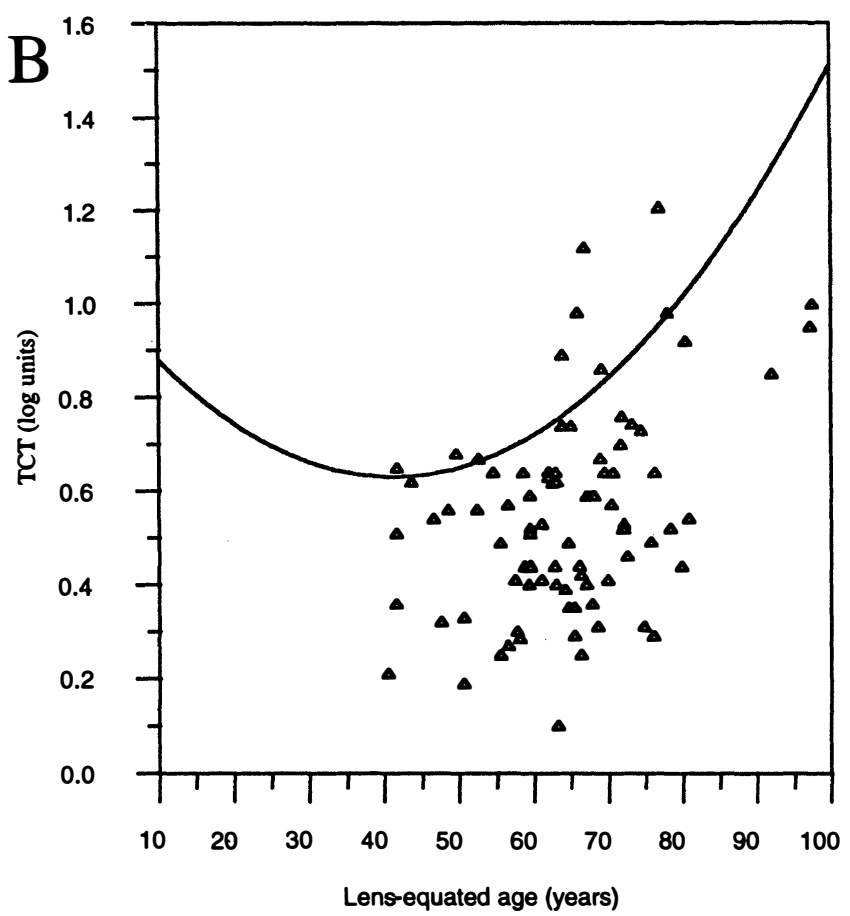

Fig. 2. Scatterplots for both RGCT $(A)$ and TCT $(B)$ estimates obtained from 87 diabetic eyes classified as having no diabetic retinopathy plotted against 'lens-equated' age. The continuous curve represents the respective 2 standard deviation limit of normality. Estimates which fall below the curve should be considered as normal. 
estimates plotted against 'lens-equated' age. When comparisons were made of both the TCT and RGCT data between the no-retinopathy group and the 'lensequated' controls using a two-sample $t$-test, it turned out that no significant differences could be found for either TCT or RGCT (TCT: $t=0.182, p=0.856$; RGCT: $t=0.547, p=0.586)$.

A two-sample comparison between diabetic eyes classified as having background retinopathy $(n=116)$ and age-matched control eyes showed that both TCT and RGCT estimates are significantly higher in the background group (TCT: $t=9.16, p<0.0001$; RGCT: $t=5.26, p<0.0001)$. A similar two-sample comparison between diabetic eyes categorised as having background retinopathy and a corresponding group of 'lens-equated' control eyes also showed that both TCT and RGCT estimates were significantly higher in the background group (TCT: $t=7.02, p<0.0001$; RGCT: $t=3.30, p<0.001)$.

The fact that background retinopathy, in the absence of macular oedema or ischaemia, had a deleterious effect on central colour vision, even after accounting for the effect of the lens, is interesting. It is possible that damage may have occurred at the macula in some of the background retinopathy eyes due to signs of macular oedema or ischaemia being unrecognised. If this was the case, one might expect that those diabetic eyes originally classified as having background retinopathy with abnormal colour vision (after compensation for lens absorption) would develop clinically significant macular oedema or ischaemia in the near future.

To test this hypothesis, all diabetics whose eyes were originally classified as having background diabetic retinopathy were recalled for fundus reassessment 18 months after their original assessment. $^{20,25}$ Of the 116 eyes originally categorised as having background diabetic retinopathy, we reassessed 87 . The remaining 29 eyes were not reassessed due to death (7 cases), moving out of the district (8 cases) and repeated non-reattendance (14 cases).

A normal/abnormal criterion was established so that a TCT or RGCT estimate was said to be abnormal if it was 2 or more standard deviations above the 'lens-equated' group-matched mean. Based on this criterion, 19 of the 87 background retinopathy eyes had abnormal TCT estimates on initial assessment and 11 of the 87 background retinopathy eyes had abnormal RGCT estimates on initial assessment.

Of the 19 eyes which originally had an abnormal TCT estimate, 12 (63\%) developed either clinically significant macular oedema or ischaemia whereas, of the remaining 68 eyes, which originally had a normal TCT estimate, only $6(9 \%)$ developed clinically significant macular oedema or ischaemia. Of the 11
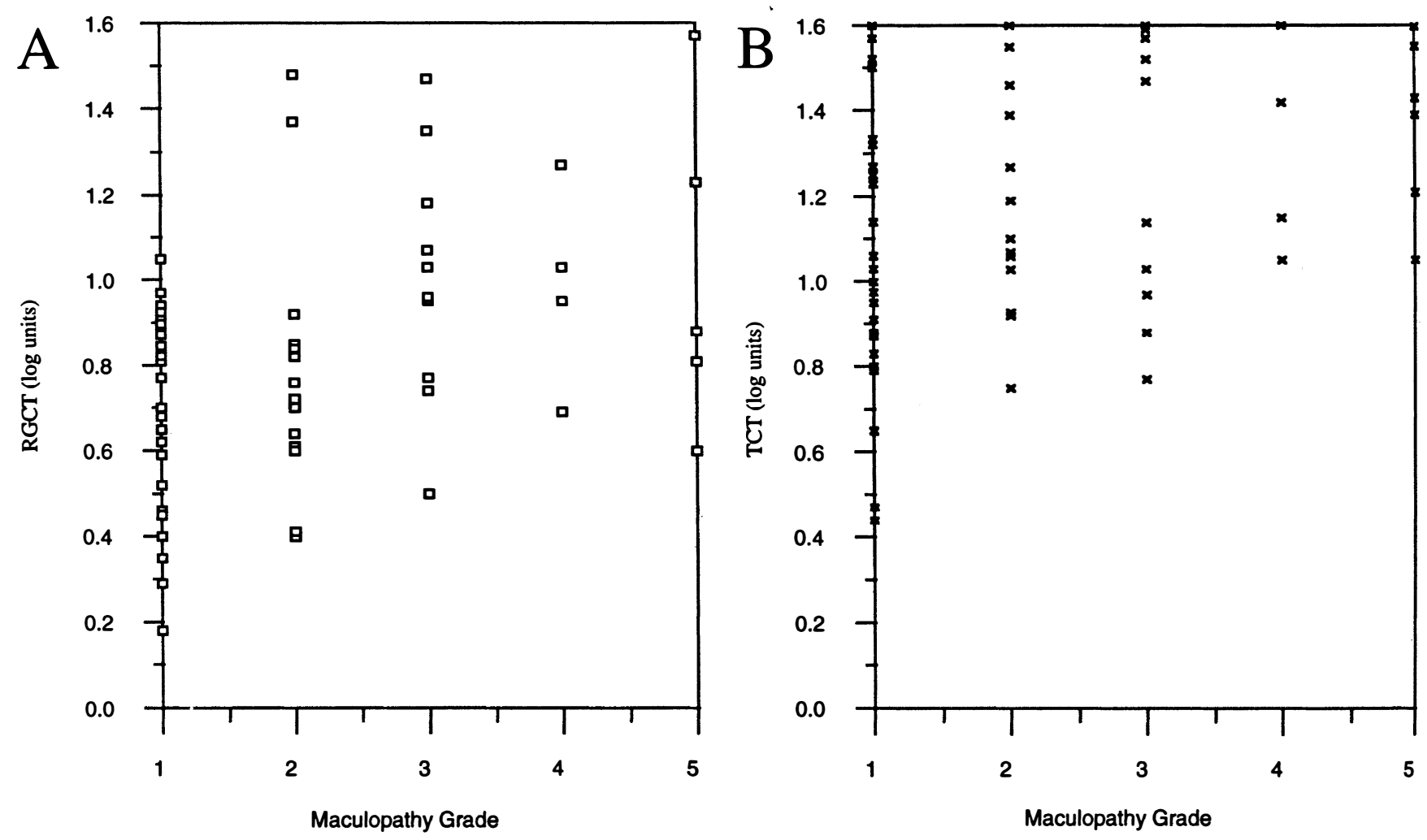

Fig. 3. Scatterplots showing RGCT $(A)$ and $T C T(B)$ versus grade of maculopathy. Spearman's rank correlation analysis found that a deterioration in both RGCT and TCT is associated with severity of maculopathy. Note that the TCT data are effectively capped due to the severity of the losses seen in eyes with maculopathy which resulted in some patients being unable to see any of the tritan stimuli presented by the SGM. 
eyes which originally had an abnormal RGCT estimate, $6(55 \%)$ developed significant macular oedema or ischaemia whereas $12(18 \%)$ of the remaining 76 eyes originally having normal RGCT estimates developed clinically significant macular oedema or ischaemia. A chi-squared analysis of the data found that those diabetics with background diabetic retinopathy and an abnormal TCT estimate or RGCT estimate are significantly more at risk of developing sight-threatening diabetic retinopathy within 18 months of the test (chi-squared, $p<0.0001$ for both TCT and RGCT estimates).

A two-sample comparison between diabetic eyes classified as having maculopathy $(n=63)$ and agematched normal eyes $(n=63)$ found that both TCT and RGCT estimates were significantly higher in the maculopathy group (TCT: $t=14.89, \quad p<0.0001$; RGCT: $t=8.85, p<0.0001)$. A similar comparison between diabetic eyes categorised as having maculopathy and a corresponding group of 'lens-equated' eyes also found that both TCT and RGCT estimates were significantly higher in the maculopathy group (TCT: $t=13.00, \quad p<0.0001 ; \quad$ RGCT: $t=8.3854$, $p<0.0001)$.

A multiple-regression analysis of the maculopathy data found a significant relationship between TCT estimate and age at onset of diabetes $(t=2.57$, $p<0.05)$. No such association was found between TCT estimate and duration of disease, presence of hard exudates within $500 \mu \mathrm{m}$ of the fovea, or presence of microaneurysms within $500 \mu \mathrm{m}$ of the fovea. An identical multiple-regression analysis using RGCT as the dependent variable found no significant association between RGCT and age at onset, duration of disease, presence of hard exudates within $500 \mu \mathrm{m}$ of the fovea, or presence of microaneurysms within $500 \mu \mathrm{m}$ of the fovea.

Data obtained from eyes with maculopathy were further subdivided into one of five groups which were categorised according to their degree of macular oedema. A subject with minimal macular oedema was graded as grade 1 and a subject with cystoid macular oedema was graded as grade 5. Any diabetic eyes showing evidence of ischaemia were excluded from this analysis. Fig. 3 shows the distribution of both TCT and RGCT estimates with increase in macular oedema severity. A Spearman's rank correlation test of the data shows that both TCT and RGCT increase with increasing macular oedema grade (TCT: $r_{\mathrm{Sp}}=0.37, p<0.005$; RGCT: $r_{\mathrm{Sp}}=0.36$, $p<0.01)$.

A two-sample comparison between diabetic eyes classified as having ischaemia $(n=33)$ and agematched control eyes $(n=33)$ found that both TCT and RGCT estimates were significantly higher in the ischaemic eyes (TCT: $t=9.41, p<0.0001$; RGCT: $t=$ $7.23, p<0.0001)$. A similar comparison between diabetic eyes categorised as having ischaemia and a corresponding group of 'lens-equated' control eyes also found that both TCT and RGCT estimates were significantly higher in the ischaemic group (TCT: $t=$ 6.42, $\mathrm{p}<0.0001$; RGCT: $t=5.21, p<0.0001)$.
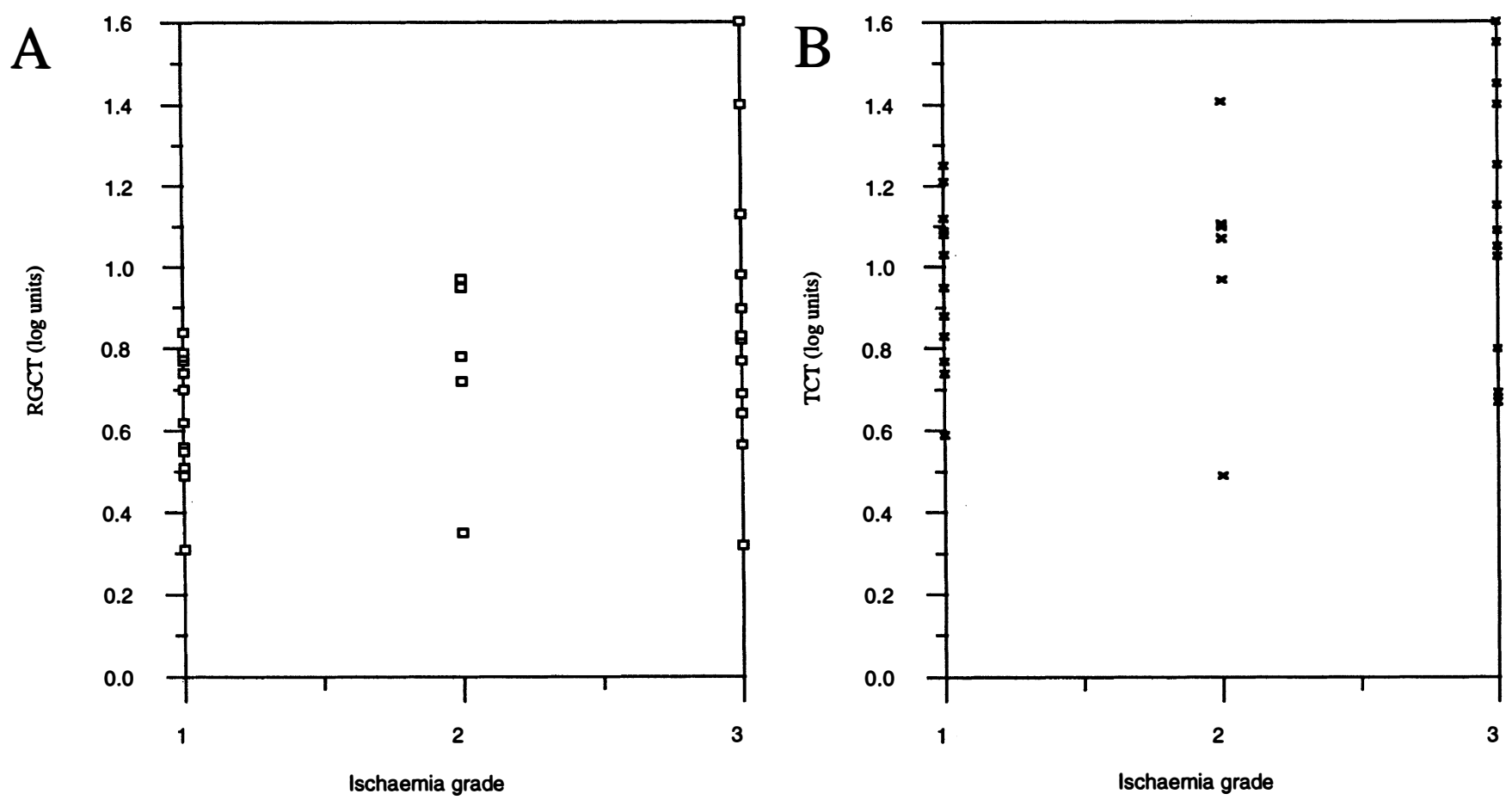

Fig. 4. Scatterplots showing RGCT $(A)$ and TCT $(B)$ versus the grade of ischaemia. It can be seen that both $R G C T$ and TCT tend to increase with increased severity of ischaemia. Spearman's rank correlation analysis found that there was a tentative association between RGCT and severity of ischaemia. However, no significant association between TCT and ischaemic severity could be found (see text). 
A multiple-regression analysis of the data obtained from the ischaemic eyes found that neither age nor age at onset showed an association with either TCT or RGCT increase. The same multiple-regression analysis of the ischaemic data showed that neither TCT nor RGCT were affected by the presence of new vessels.

Data obtained from the ischaemic eyes were further subdivided into mild (grade 1) ischaemia, moderate (grade 2) ischaemia and severe (grade 3) ischaemia. This further classification of ischaemic eyes was based on the number of ischaemic features present on the fundus. For example, an eye with only a few cotton-wool spots was classified as grade 1 . An eye with many cotton-wool spots, deep retinal haemorrhages, intraretinal microvascular abnormalities and signs of venous changes was classified as grade 2. An eye which had peripheral or disc new vessels was classified as grade 3 . It should be noted that in this study the degree of ischaemia was not judged on the results of fluorescein angiography. Fig. 4 shows that increases in both TCT and RGCT estimates seem to be associated with the degree of ischaemia. However, Spearman's rank correlation test found only a tentative association between an increase in RGCT and increased severity of ischaemia, and no such association was found for TCT and severity of ischaemia (TCT: $r_{\mathrm{Sp}}=0.19, p=0.25$; RGCT: $\left.r_{\mathrm{Sp}}=0.32, p=0.05\right)$.

\section{DISCUSSION}

The results reported here confirm that colour vision as determined by chromatic-contrast threshold estimates, especially TCT estimates, is affected by diabetes. This study has shown that the principal factors which affect colour vision in the diabetic eye are age at onset, duration of disease, the presence and degree of macular oedema and, finally, the presence of retinal ischaemia. These findings are in agreement with the earlier work of Reffin ${ }^{16}$ and de Alwis ${ }^{17}$ who also found these same factors to have a significant effect on colour vision as determined by the SGM. Whilst significant losses in colour vision were seen in the great majority of diabetic eyes with retinopathy classified as being sight-threatening, it is interesting to note that colour vision was also reduced in some diabetic eyes which either showed no evidence of retinopathy or were classified as having mild, non-sight-threatening retinopathy.

The results reported in this paper show that patients with no clinical evidence of retinopathy tend to have reduced TCT estimates and normal RGCT estimates, indicating a selective tritan colourvision loss. Several other studies into colour-vision changes in diabetics with no retinopathy have also identified a selective tritan loss. For example, Kinnear et al. ${ }^{7,8}$ found, using the Pickford-Nicholson anomaloscope, that a tritan loss was the most common form of colour-vision loss in their noretinopathy diabetic population. Zisman and Adams $^{26}$ used an electroretinographic technique to demonstrate a reduced S-cone pathway sensitivity in type I diabetics without clinically observed retinopathy.

On further analysis of the data obtained from type I diabetics with no retinopathy we found that the tritan loss was duration dependent, and when the data were corrected for the effects of durationdependent lens yellowing the specific tritan loss disappeared. This finding lends support to the hypothesis that the relative reduction in tritan colour vision seen in diabetics with no retinopathy is due to lens yellowing, and not to selective damage to the tritan colour-vision system caused by microscopic retinal changes. These results are contrary to those reported in a recent paper by Hardy et al. ${ }^{27}$ who compared the FM 100-hue error scores obtained from 25 type I diabetics with no retinopathy as determined by fluorescein angiography with those of 25 'lens-equated' controls. They concluded that some, but not all the losses seen in diabetics with no retinopathy could be explained by lens changes.

If one accepts that duration-dependent effects are responsible for some of the tritan colour-vision losses seen in diabetics, then it is reasonable to argue that the relatively greater increase in all the diabetic groups of TCT compared with RGCT is, in fact, due to the selective filtering out of short-wavelength light by the yellowed lens rather than to an increased susceptibility of the S-cone colour-vision channel to retinal damage. Indeed, de Alwis ${ }^{17}$ found that when one adjusted for the effects of duration as determined by multiple-regression analysis on colour vision, the relative diferences between RGCT and TCT across all retinopathy groups disappeared. A similar analysis of the data obtained from our type I diabetics of all retinopathy grades produced identical results. This would indicate that the diabetic retinopathy itself affects both red/green- and tritancontrast threshold equally and that the relative increase in tritan-contrast threshold, when compared with red/green-contrast threshold, is, in fact, due to the superimposition of the effects on tritan-contrast threshold due to lens yellowing. It should be noted that one cannot, on the basis of these results alone, disregard the possibility that microscopic changes in the retina are indeed selectively damaging the S-cone system and hence reducing the sensitivity of the tritan colour-vision system. However, one should consider, a little more sceptically than might otherwise be the case, reports which claim that tritan colour-vision is more susceptible than red/green colour vision to the effects of retinal damage due to diabetes. 
Another important point demonstrated by the results presented in this paper is that those subjects with background retinopathy who have abnormal chromatic-contrast thresholds (after compensation for lens yellowing) are at a higher risk of developing clinically significant sight-threatening retinopathy than those who have chromatic-contrast thresholds which fall within the normal range. It is possible that had fluorescein angiography been carried out on those subjects who had abnormal colour vision, many of them would have turned out to have sightthreatening diabetic retinopathy which was undetectable with a slit lamp. Unfortunately, it was not possible for us to carry out such a study for ethical reasons, since fluorescein angiography is an invasive technique which carries a small, but significant, risk to the subject. Despite this, it is obvious that measurement of chromatic-contrast thresholds, particularly along a tritan confusion axis, using a sensitive clinical testing system such as the SGM, is useful in predicting which diabetics with non-sightthreatening retinopathy are likely to suffer a sightthreatening form of retinopathy within a relatively short period of time. This finding is of profound clinical importance since it shows that measurement of chromatic-contrast thresholds can provide a powerful non-invasive approach by which one can monitor those diabetics with non-sight-threatening retinopathy and flag those who are most at risk of deteriorating to a sight-threatening stage, thus allowing early treatment as soon as treatable lesions develop.

Previous reports by other authors support our finding that colour vision deteriorates in the presence of maculopathy. ${ }^{10,13,14,16,17,28}$ Bresnick et al ${ }^{14}$ found that the magnitude of error score as determined by the FM 100-hue test was associated with degree of macular oedema. Green et al. ${ }^{10}$ reported that, in their study, $95 \%$ of the diabetic eyes with maculopathy had abnormal vision. Both Bresnick et al. ${ }^{14}$ and Greenstein et al. ${ }^{28}$ also showed that the FM 100-hue error score increased with the severity of macular oedema. Reffin ${ }^{16}$ and de Alwis ${ }^{17}$ found that no association existed between the presence of hard exudates or microaneurysms within $500 \mu \mathrm{m}$ of the fovea and colour-vision loss. However, our results differ from those of Reffin and de Alwis in that the duration of disease showed no measurable effect on either TCT or RGCT in those diabetics with clinically significant maculopathy. It is possible that the effects of maculopathy on colour vision are so severe that any relationship between duration and colour-vision deterioration is masked. However, the fact that age at onset was significantly associated with an increase in TCT indicates that the duration estimates in this group of diabetics, who are predominantly type II, are poor and that much of the effect of duration on tritan colour vision is hidden within the age-at-onset variable.

Unfortunately, many of the subjects in this study with macular oedema had colour-vision losses which were so severe that they could not see the stimuli at all. This problem, which is common to all CRT-based colour-vision testing systems, only occurred with the TCT data and one must accept that the regression analysis gives us little more information than that there is indeed an increase in TCT with increase in macular oedema severity. The magnitude of the increase cannot be determined since the data have effectively been capped.

It is possible that had fluorescein angiography been used to better classify the ischaemic eyes in this study, the classification of the degree of ischaemia would have been more accurate and perhaps an association between increase in chromatic-contrast threshold and the degree of retinal ischaemia might have been found. Reffin and de Alwis have reported that a significant relationship does exist between the ischaemic grade, as determined by ophthalmoscopy, and an increase in both TCT and RGCT estimates as determined with the SGM. ${ }^{16,17}$ As a result it is advisable that the reader treat with caution the results reported here regarding the effects of ischaemic severity on colour vision. Further studies using a more stringent protocol need to be carried out to clarify the situation.

The finding that the presence of new vessels seemed to have no effect on chromatic contrast is in agreement with those of Reffin and de Alwis who also found that the presence of new vessels had no effect on colour vision. ${ }^{16,17}$ This is not a surprise since new-vessel growth is a response to severe ischaemia of the retina. It would seem logical to doubt that new vessels on their own would have any effect on visual function except when they haemorrhage or are responsible for a tractional retinal detachment.

Finally, the fact that colour vision seems to be so intimately associated with diabetes lays opens the possibility that chromatic-contrast testing might provide the clinician with a non-invasive technique for screening for sight-threatening diabetic retinopathy. We are at present carrying out a 2 year prospective study at the Sussex Eye Hospital, in conjunction with the Royal Sussex County Hospital diabetic and medical photography departments, aimed at determining whether a role does exist for chromatic-contrast testing in screening the diabetic population for sight-threatening diabetic retinopathy. We envisage that automated chromatic-contrast threshold testing systems such as the SGM will supplement other screening procedures such as fundus photography and that the two modalities will provide an overall screening system that will not only effectively detect those with sight-threatening 
forms of diabetic retinopathy, but will also make it possible to flag those diabetics with seemingly mild forms of retinopathy, or no retinopathy at all, who are most likely to develop sight-threatening retinopathy in the relatively near future.

Key words: Diabetic retinopathy, Colour vision, Tritan, Redgreen, Chromatic contrast.

\section{REFERENCES}

1. Zanen J. Introduction à l'étude des dyschromatopsias rétiennes contrales acquises. Bull Soc Belg Ophtalmol 1953;103:3-148.

2. Dubois-Poulsen A, Cochet P. Un cas de dyschromatopsie diabétique. Bull Soc Ophtalmol France 1954; 4:323-30

3. Cox J. Colour vision defects acquired in diseases of the eye. Br J Physiol Opt 1960;17:195-216.

4. Hong S. Types of acquired colour-vision defects. Arch Ophthalmol 1957;58:505-9.

5. Verriest G. Further studies on acquired deficiency of colour discrimination. J Opt Soc Am 1963;53:185-95.

6. Verriest G. Les déficiences acquises de la discrimination chromatique. Mem Acad R Med Belg 1964;5: 325-7.

7. Kinnear PR, Aspinall PA, Lakowski R. The diabetic eye and colour vision. Trans Ophthalmol Soc UK 1972;92:69-78.

8. Lakowski R, Aspinall PA, Kinnear PR. Association between colour vision losses with diabetes mellitus. Ophthalmic Res 1972;4:145-9.

9. Trick GL, Burde RM, Gordon MO, Santiago JV, Kilo C. The relationship between hue discrimination and contrast sensitivity deficiencies in patients with diabetes mellitus. Ophthalmology 1988;95:693-8.

10. Green FD, Ghafour IM, Allan D, Barrie T, McClure E, Foulds WS. Colour vision in diabetes. Br J Ophthalmol 1985;69:533-6.

11. Lombrail P, Gervais P, Cathelineau G. Prediction of diabetic retinopathy from colour vision data. Diabetes Care 1983;6:621-2.

12. Roy MS, Gunkel RD, Podgor MJ. Colour vision defects in early diabetic retinopathy. Arch Ophthalmol 1986;104:225.

13. Roy MS, McCullough C, Hanna AK, Mortimer C. Colour vision in long standing diabetes mellitus. $\mathrm{Br} \mathrm{J}$ Ophthalmol 1984;68:215-7.

14. Bresnick GH, Condit R, Palta M. Association of huediscrimination loss and diabetic retinopathy. Arch Ophthalmol 1985;103:1317-24.
15. Utku D, Atmaca LS. Farnsworth-Munsell 100 hue test for patients with diabetes mellitus. Ann Ophthalmol 1992;24:205-8.

16. Reffin JP. The design and clinical application of tests of colour vision. DPhil Thesis, University of Sussex, Falmer, Brighton, 1992.

17. de Alwis DV. The role of automated chromatic contrast sensitivity tests in the management of diabetic retinopathy. MPhil Thesis, University of Sussex, Falmer, Brighton, 1994.

18. Early Treatment Diabetic Retinopathy Study Research Group. Photocoagulation for diabetic macular oedema. Early Treatment Diabetic Retinopathy Study report no. 1. Arch Ophthalmol 1985;103:1796-806.

19. Early Treatment Diabetic Retinopathy Study Research Group. Treatment techniques and clinical guidelines for photocoagulation of diabetic macular oedema. Early Treatment Diabetic Retinopathy Study report no. 2. Ophthalmology 1987;94:761-4.

20. Tregear SJ, Knowles PJ, Ripley LG, Casswell AG. Colour vision deficits predict the development of severe diabetic retinopathy in diabetic subjects with background retinopathy. Invest Ophthalmol Vis Sci (ARVO Abstr) 1993;34:S719.

21. Cornsweet TN. The staircase method in psychophysics. Am J Psychol 1962;75:485-91.

22. Lutze M, Bresnick G. Lenses of diabetic patients 'yellow' at an accelerated rate similar to older normals. Invest Ophthalmol Vis Sci 1991;32:194-9.

23. Moreland JD. Lens equated age controls for diabetics. Invest Ophthalmol Vis Sci 1993;34:281-2.

24. Pokorny J, Smith VC, Lutze M. Aging of the human lens. Appl Optics 1987;26:1437-40.

25. Tregear SJ, Ripley LG, Knowles PJ, Gilday RT, de Alwis DV. Automated tritan discrimination: a new clinical technique for the effective screening of severe diabetic retinopathy. Int J Psychophys 1994;16:191-8.

26. Zisman F, Adams AJ. Spectral sensitivity of cone mechanisms in juvenile diabetics. Doc Ophthalmol Proc Ser 1982;32:127-31.

27. Hardy KJ, Scarpello JHB, Foster DH, Moreland JD. Effect of diabetes associated increases in lens optical density on colour discrimination in insulin dependent diabetes. Br J Ophthalmol 1994;78:754-6.

28. Greenstein V, Sarter B, Hood D, Noble K, Carr R. Hue discrimination and s-cone pathway sensitivity in early diabetic retinopathy. Invest Ophthalmol Vis Sci 1990;31:1008-14. 